\title{
Mitigation of nonlinear effects through frequency shift free mid-span spectral inversion using counter-propagating dual pumped FWM in fiber
}

\author{
Abhishek Anchal ${ }^{1}$, Pradeep Kumar ${ }^{1,2}$ and Pascal Landais ${ }^{3}$ \\ Department of Electrical Engineering, Indian Institute of Technology Kanpur, Kanpur, 208016, India \\ Center for Laser and Photonics, Indian Institute of Technology Kanpur, Kanpur, 208016, India \\ School of Electronic Engineering, Dublin City University, Glasnevin, Dublin 9, Ireland
}

Abstract

We propose and numerically verify a scheme of frequency-shift free mid-span spectral inversion (MSSI) for nonlinearity mitigation in an optical transmission system. Spectral inversion is accomplished by optical phase conjugation, realized by counter-propagating dual pumped four-wave mixing in a highly nonlinear fiber. We examine the performance of MSSI due to critical parameters such as nonlinear fiber length, pump and signal power. We demonstrate the near complete nonlinearity mitigation of 40 Gbps DQPSK modulated data transmitted over $1000 \mathrm{~km}$ standard single mode fiber using our method of MSSI. We perform simulation of bit-error rate as a function of optical signal to noise ratio to corroborate the effect of frequency-shift free MSSI. 


\section{Introduction}

The increased demand of capacity for future optical com-munication system can be meet by advance modulation for-mats such as QPSK, 16- and 64- QAM etc [1]. However, these formats require higher optical signal-to-noise ratio (OSNR) at the receiver end compared to the simpler OOK format for the same transmission length, resulting in higher launch powers $[1,2]$. The higher launch power, coupled with small core area of standard single-mode transmission fiber (SSMF), exacerbates the nonlinear effects and causes the distortion of signal leading to increased bit error rate (BER) and reduced transmission reach [3]. A variety of techniques are used in practice to mitigate the nonlinear effects in fiber, including digital signal processing based back propagation and all-optical solutions such as; phase-conjugated twin waves, phase sensitive amplification, and mid-span spectral inversion (MSSI) [4-8].

Among these, MSSI using optical phase conjugation (OPC) is one technique that can fully compensate distortions due to fiber nonlinearities and dispersion [7, 8]. OPC is transparent to modulation format and can be implemented entirely in the optical domain [9, 10]. In addition, OPC based MSSI can be used for simultaneous multi-channel compen-sation of distortions induced by fiber nonlinearity and dis-persion [11, 12]. MSSI using OPC was first proposed and experimentally realized by Yariv et al, in which inversion of distortion due to nonlinearities and dispersion in the first half of transmission fiber by mid link OPC, is cancelled out by similar nonlinear distortion in the second half of the fiber [13]. MSSI based on optical-electrical-optical conversion was proposed for nonlinear compensation [14]. However, in this method, spectral inversion adds additional delay due to optical-electrical-optical conversion process.

Nonlinearity mitigation based on MSSI using OPC has been demonstrated by four-wave mixing (FWM) in single mode fiber and semiconductor optical amplifiers (SOA) [7, 15, 16]. However, frequency conversion during the pro-cess of MSSI by OPC leads to crosstalk in WDM system, and thus increases the BER. Several techniques for generation of frequency-shift free MSSI has been reported, which include a co-propagating orthogonally polarized dual pump FWM in SOA or fiber $[17,18]$. However, these techniques require the controlling of the polarization of signal, in a way that pumps are co- and orthogonally-polarized with signal. The resulting conjugate experiences a shift of polarization with respect to signal. Since, it is difficult to readily control the polarization signal in a transmission system, a frequency and polarization-shift free MSSI is required [17].

In this paper, we elaborate on our previously reported technique of polarization and frequency-shift free OPC in highly nonlinear fiber (HNLF) [19] and investigate its per-formance as MSSI for nonlinearity compensation of 40 Gbps DQPSK modulated optical signal transmitted over $1000 \mathrm{~km}$ standard single-mode fiber (SSMF). MSSI is comprised of polarization and frequency-shift free OPC emanates from counter-propagating dual pump FWM in HNLF. In this scheme two counter-propagating pumps along with a signal co-propagating with one of the pumps, creates two Bragg gratings, which reflects the other pump to generate a con-jugate wave counterpropagating with signal $[9,17]$.

We verify the dependence of efficiency and phase-conjugation of idler on signal power. Further, we investigate the performance of frequency-shift free OPC as MSSI by predicting divergency of bit-error rate (BER) on the HNLF length, pump power, and signal power. Later we verify nonlinearity com-pensation using proposed MSSI by investigating BER perfor-mance of system optical signal-to-noise ratio (OSNR).

This paper is organized as follows: in section 2, we discuss frequency-shift free MSSI using counterpropagating dual pump FWM in HNLF. In section 3, we scrutinize MSSI performance by studying BER variation with different OPC parameters, signal power and transmitted OSNR for DQPSK transmission. Finally, in section 4 , we conclude by summar-izing our results.

\section{System description}

The schematic of a frequency-shift free MSSI-based trans-mission link is shown in the figure 1. At the transmitter, symbols $d_{n} €\{1,+j,-1,-j\}$ are DQPSK encoded onto the optical carrier of the angular frequency $\omega_{s} \mathrm{rad} / \mathrm{s}$, emitted by the laser diode. The modulated signal is launched into the first half of the transmission fiber where it accumulates dispersion and nonlinearity induced phase. This phase is conjugated by the OPC placed half-way between the transmitter and receiver as depicted in figure 1 . The phase conjugated signal is then transmitted through second half of the fiber, where its con-jugated phase is corrected in the second half of the fiber. The received signal is coherently detected and processed to recover the transmitted symbols. The BER is obtained by error counting the demodulated sequence. In order to visualize only nonlinearity mitigation by MSSI, we compensate chromatic dispersion using in-line DCFs; it is also possible to employ MSSI itself to compensate dispersion. 
The MSSI block is shown in the inset of figure 1. The input amplifier EDFA 1 boosts the transmitted power and band-pass filter $\mathrm{BPF}_{1}$ removes out of band amplified spontaneous emission (ASE) noise. The amplified signal $\left(\omega_{S}\right)$ then combined with pump1 $(\omega 1)$ and fed into one end of HNLF. Pump2 $\left(\omega_{2}\right)$ is launched into other end of HNLF. These three waves interact via FWM process in HNLF and generate the backward propagating idler at signal band, whose phase is conjugate of signal phase. However, in this process the exchange of power between two pumps, pump1 and pump2, causes the FWM instability [21, 22]. This FWM instability is due to the powerdifference between the two pumps [23]. The exchange of power between pumps can be written as (it is referenced as equation (5) in [21]),

$$
\Delta P=\gamma^{2} L^{2} P_{1} P_{2}\left(P_{1}-P_{2}\right),
$$

where $\mathrm{P}_{1}$ and $\mathrm{P}_{2}$ are the power of pump1 and pump2 respectively. When (a) $P_{1}>P_{2}, \Delta P>0$, power is transferred from pump1 to pump2, (b) $P 1<P_{2}, \Delta P<0$, power is transferred from pump2 to pump1, and (c) $P 1=P 2, \Delta P=0$, no power is transferred. Therefore, in MSSI scheme, we keep the two pump powers equal to avoid the FWM instability.

The backward propagating conjugated signal is passed through optical tunable filter to remove the pump2. The dotted box in inset of figure 1 shows optical phase conjugation. The phase conjugated signal is then amplified by EDFA2$\mathrm{BPF}_{2}$ system before passing to the receiver. The function of $\mathrm{BPF}_{2}$ is similar to the $\mathrm{BPF}_{1}$. The spectrum of signal, pumps and conjugate at two ends of HNLF are shown in the figure 1. Spectral inversion by MSSI is shown in the conjugate band.

\subsection{Performance of OPC}

We study the performance of OPC under the effect of signal power. The dependency of conjugate on HNLF length and pumps power are studied in our previous work [19]. The typical parameters used in the simulation are: pump wave-lengths $\lambda_{1}=1539.5 \mathrm{~nm}$ and $\lambda_{2}=1540.5 \mathrm{~nm}$, signal and idler wavelengths $\lambda_{s}(=\lambda i)=1540 \mathrm{~nm}$, signal and pumps power of $-7 \mathrm{dBm}$ and $9.22 \mathrm{dBm}$ respectively. The para-meters from commercially available HNLF are used in the simulation: zero dispersion wavelength of $1540 \mathrm{~nm}, \gamma=9.3 / \mathrm{W} / \mathrm{km}$, and dispersion slope $=0.071 \mathrm{ps} /\left(\mathrm{nm}^{2}\right.$ $\mathrm{km})$ [24]. By keeping two pumps power equal, we reduce the FWM instability due to the exchange of power between two pumps [21, 22]. We allow detuning between pumps and signal $\Omega=63.25 \mathrm{GHz}$ to avoid the effects of stimulated Brillouin and Raman scattering, considering the fact that these scattering processes have a significant gain at 10.45 $\mathrm{GHz}-10.55 \mathrm{GHz}$ and $5 \mathrm{THz}-20 \mathrm{THz}$ detuning range respectively.

Effect of signal power: We study the effect of signal power on the efficiency and phase-offset by keeping the pumps power constant at $9.22 \mathrm{dBm}$ at HNLF length of 0.95 Lop. Figure 2(a) shows that efficiency is constant with the increasing signal power for $\alpha=0$. This efficiency decreases considerably when attenuation $\alpha=0.9 \mathrm{~dB} / \mathrm{km}$ is included in the simulation. This is due to attenuation of pumps and signal powers. When we considered SPM and XPM effects, efficiency decreases as compared to efficiency when $\alpha=0$, this is due to the increase in residual phase mismatch | Dk| due to SPM and XPM of signal phase by signal, pump1 and pump2 beams. This increases the residual phase mismatch further with the increased signal power and causes reduction in efficiency, in our case this occurs at $-5 \mathrm{dBm}$ signal power. The efficiency is further reduced, when $\alpha=0.9 \mathrm{~dB} / \mathrm{km}$ and SPM and XPM effects are taken into account, resulting in $-17 \mathrm{~dB}$ of efficiency at $-7 \mathrm{dBm}$ of signal power $9.22 \mathrm{dBm}$ of pump powers for HNLF length of $0.95 \mathrm{Lop}$.

Figure 2(b) shows the effect of signal power on the phase-offset $\Delta \phi$ offset. The phase-offset is nearly zero till $-5 \mathrm{dBm}$ signal power for both theory and simulations at $\alpha=0$ for $\mathrm{L}=\mathrm{Lop}$ and at $\alpha=0.9 \mathrm{~dB} / \mathrm{km}$ with SPM and XPM for respectively, showing phase conjugation. A L=0.95Lop non-zero phase-offset is present at all other powers for $\mathrm{a}=0$ at $\mathrm{L}=0.95 \mathrm{Lop}$. With $\alpha=0.9 \mathrm{~dB} / \mathrm{km}$, signal and pump power decreases which results in an increase in phase offset, whereas, when SPM and XPM effects are considered with $\alpha=0$, phase-offset decreases due to increase in residual phase mismatch $|\Delta \mathrm{k}|$. When the effects of attenuation and dispersion-compensating fiber (Length $=200 \mathrm{~km}, \alpha d c f=0.5 \mathrm{~dB} / \mathrm{km}$ and $D=-80$ $\mathrm{ps} / \mathrm{nm}-\mathrm{km}$ ) for inline dispersion compensation. OPC parameters are taken same as mentioned in section 2.2. We have used matlab toolbox Optilux to simulate DQPSK transmission [25]. Previous study was carried using QPSK in a similar network at the same data rate [26].

We compute the BER performance of system as a function of MSSI parameters: HNLF length and pump power. We preserve the same simulation strategies as mentioned in section 2.2 i.e., first consider ideal MSSI system without attenuation $(\alpha=0)$ and SPM and XPM effects in HNLF and then introduce $\alpha=0.9 \mathrm{~dB} / \mathrm{km}$ and 
SPM and XPM effects in steps. Finally we combine the two effects to visualize the effect of MSSI on BER of transmission system.

\subsection{Influence of fiber length}

Figure 3 shows BER value of system with different HNLF length, for signal power of $-7 \mathrm{dBm}$ and OPC parameters, $\alpha=0$, pumps power of $9.22 \mathrm{dBm}$. BER decreases with HNLF length till $\mathrm{L}_{\mathrm{op}}$ and then increases after $\mathrm{L}_{\mathrm{op}}$, thus giving a minimum value of $1 \times 10^{-9}$ at optimum HNLF length. The BER varies periodically with HNLF length with periodicity $2 \mathrm{Lop}$. When attenuation $\alpha=0.9 \mathrm{~dB} / \mathrm{km}$ is included into simulation, BER lies in the range of $10^{-7}-10^{-}$ 8 after $0.8 L_{o p}$ as phase-offset by OPC increases after $0.8 L_{o p}$.

When only SPM and XPM effects are considered in the simulation, BER increases due to an increase in phaseoffset provided by OPC and this effect increases with HNLF length, which results in further augmentation in BER. Finally, when the two effects of SPM and XPM and attenuation $(\alpha=0.9 \mathrm{~dB} / \mathrm{km})$ are combined a perfect phase conjugation is obtained at $0.95 L_{o p}$, which results in a minimum BER of $1 \times 10^{-9}$.

\subsection{Influence of pump power}

Figure 4 shows BER value of system at pump1 power of $0 \mathrm{dBm}$ to $13.83 \mathrm{dBm}$, for signal power of $-7 \mathrm{dBm}$, pump2 power of $9.22 \mathrm{dBm}$. BER decreases with increases in pump1 power as the phase-offset decreases for HNLF length of $L=L_{o p}$ with $\alpha=0$. At pump1 power of $9.22 \mathrm{dBm}$, the BER reduces to minimum value of $1 \times 10^{-9}$, due to perfect phase conjugation operation by OPC as well as stability of FWM process at equal pump power [21, 22]. When attenuation $(\alpha=0.9 \mathrm{~dB} / \mathrm{km})$ is considered into simulation for HNLF length of $L=0.95 L_{o p}$, BER shows almost constant behaviour at $10^{-7}$ as phase-offset provided by OPC is constant.

Further, when SPM and XPM effects with $\alpha=0$ at HNLF length of $L=0.95 L_{o p}$ is considered in the simulation, the BER increases due to SPM and XPM effects increasing the phase-offset provided by OPC. The offset increases with pump1 power, which results in further increase in BER. Finally, when the two effects of SPM and XPM and attenuation $(\alpha=0.9 \mathrm{~dB} / \mathrm{km})$ are combined a nearly perfect phase conjugation is obtained at $9.22 \mathrm{dBm}$ of pump1 power, which results in minimum BER of $1 \times 10^{-9}$. The transmission performance is optimised as the SPM and XPM induced penalty are reduced due to the attenuation of the signal.

\subsection{Influence of signal power}

BER as a function of transmitted signal power is shown in the figure 5 at pumps power of $9.22 \mathrm{dBm}$, HNLF length of $0.95 \mathrm{~L}_{\mathrm{op}}, \alpha=0.9 \mathrm{~dB} / \mathrm{km}$ with SPM and XPM. As signal power is increased the BER decreases, to eventually flatten at $10^{-7}$ between $-5 \mathrm{dBm}$ and $10 \mathrm{dBm}$ and increases after $10 \mathrm{dBm}$ of signal power due to higher nonlinear effects at higher signal power. When MSSI is introduced the BER is significantly reduced to $10^{-9}$ over a range of input signal from $-15 \mathrm{dBm}$ to $20 \mathrm{dBm}$.

\subsection{Influence of OSNR}

BER as a function of OSNR for transmission system with and without MSSI is plotted in figure 6. The OSNR is varied by changing noise power and keeping the signal power fixed at $-7 \mathrm{dBm}$. The BER with and without MSSI decreases with increasing OSNR. At a low transmitted OSNR, BER of the system without MSSI is as large as $10^{-2}$, whereas, after nonlinearity compensation by MSSI, BER reduces by six orders of magnitude. At high transmitted OSNR i.e. above $32 \mathrm{~dB}$, where the effect of nonlinear phase noise is low, the MSSI based configuration shows at least a two order improvement of BER with a value of $10^{-9}$.

\section{Conclusions}

In this paper, we designed a scheme of frequency-shift free MSSI for the mitigation of nonlinear effect of DQPSK modulated optical signal transmitted through SSMF. MSSI is realized using OPC in counter-propagating dual pump FWM in HNLF. Frequency conversion in conjugate wave is avoided by symmetrical placement of pump 
frequencies about signal. Appearance of conjugate and signal at opposite ends of HNLF results in natural spatial filtering and thus eliminated the needs of polarization-shift in conjugate. Further, polar-ization of signal is maintained in conjugate by keeping two pumps co-polarized.

We examine the performance of MSSI for nonlinearity compensation of DQPSK modulated signal under the effect of HNLF length and pump power. BER decreases significantly with increase in HNLF length upto HNLF length of $0.95 L_{O p}$ and increases thereafter and almost saturates in the range of $10^{-7}-10^{-8}$. A minimum BER of $1 \mathrm{x}$ $10^{-9}$ was obtained at HNLF length of $0.95 L_{o p}$. BER decreases with increasing pump1 power and minimum BER of $1 \mathrm{x}$ $10^{-9}$ was obtained at $9.22 \mathrm{dBm}$ pump1 power due to perfect phase conjugation by OPC and stable FWM process in HNLF.

Lastly, we verified the compensation of nonlinear distortion for 40 Gbps DQPSK signal transmitted over $1000 \mathrm{~km}$ fiber by frequency-shift free MSSI. BER improvement of six-order obtained at lower signal power and OSNR and more than two-order at higher signal power and OSNR confirms the significant reduction of nonlinear impairments by MSSI eliminating any pre and post processing of the transmitted data at the receiving end. In conclusion, we demonstrated mitigation of nonlinear impairments of DQPSK signal transmitted over $1000 \mathrm{~km}$ SSMF with the exploitation of OPC as frequency-shift free MSSI. Frequency shift free operation is obtained by counter- propagating dual pump FWM in HNLF. We have shown that under the right set of OPC parameters elucidated in this paper, nonlinear impairments are significantly compensated, allow-ing data transmission at a 1 $\mathrm{X} 10^{-9} \mathrm{BER}$ without pre and post data processing.

\section{Acknowledgments}

This work is supported by Science and Engineering Research Board, Department of Science and Technology, India (Grant number SERB/EE/2014161) and Marie Curie International Research Staff Exchange Scheme Fellowship within the 7th European Community Framework Programme (Grant number 318941). 


\section{Figures}

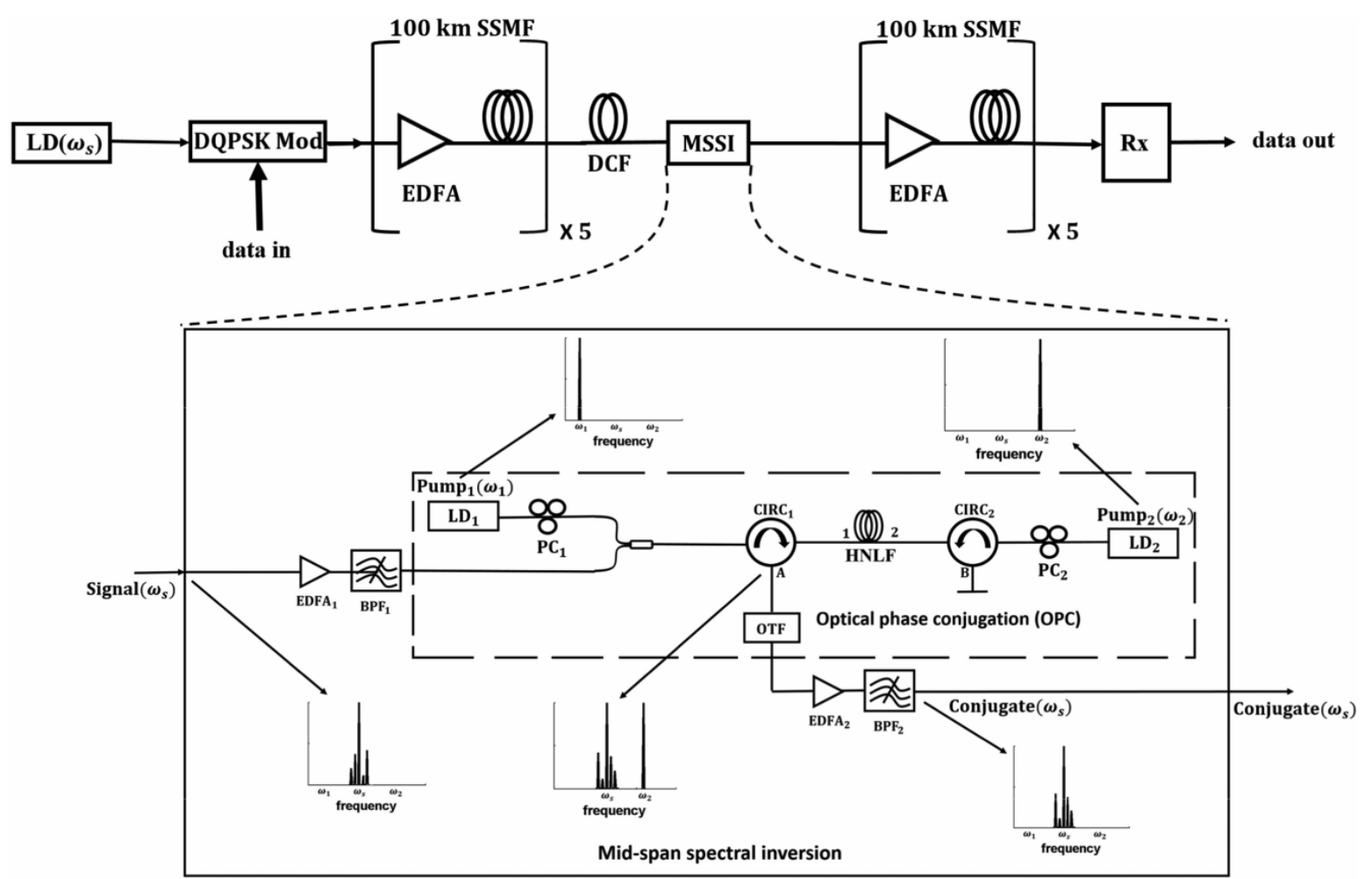

Figure 1. Proposed scheme for nonlinearity compensation of DQPSK modulated data transmitted over $1000 \mathrm{~km}$ standard single mode fiber $(\mathrm{SSMF})$. EDFA $=$ erbium doped fiber amplifier, $\mathrm{DCF}=$ dispersion compensating fiber, $\mathrm{LO}=$ local oscillator, $\mathrm{BPF} 1,2=$ band-pass filter, $\mathrm{HNLF}=$ highly nonlinear fiber, $\mathrm{PC} 1,2$ polarization controller, $\mathrm{CIRC} 1,2$ circulator, $\mathrm{LD} 1,2$ laser diode, $\mathrm{ISO}=$ isolator, $\mathrm{OTF}=$ optical tunable filter. 


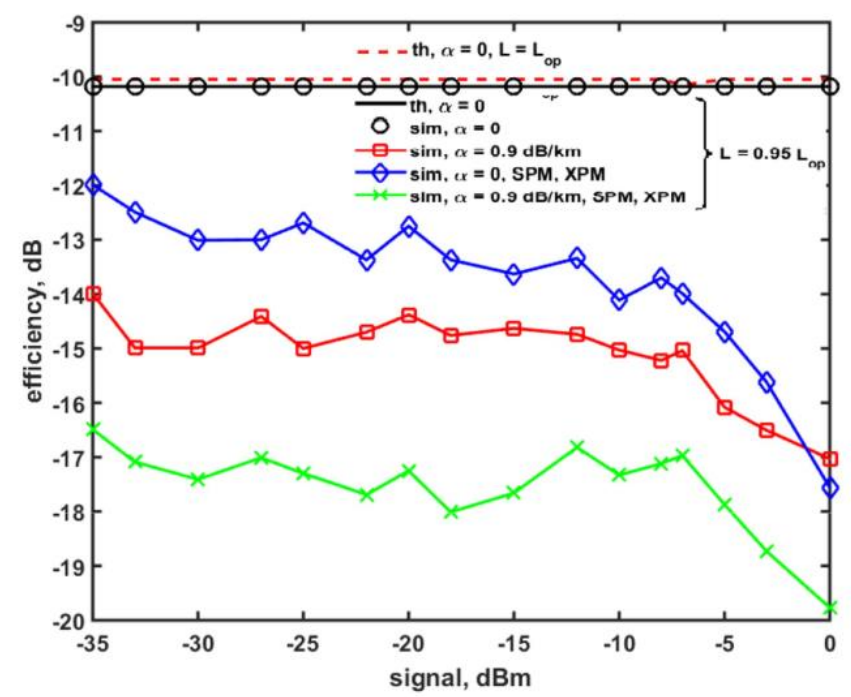

(a)

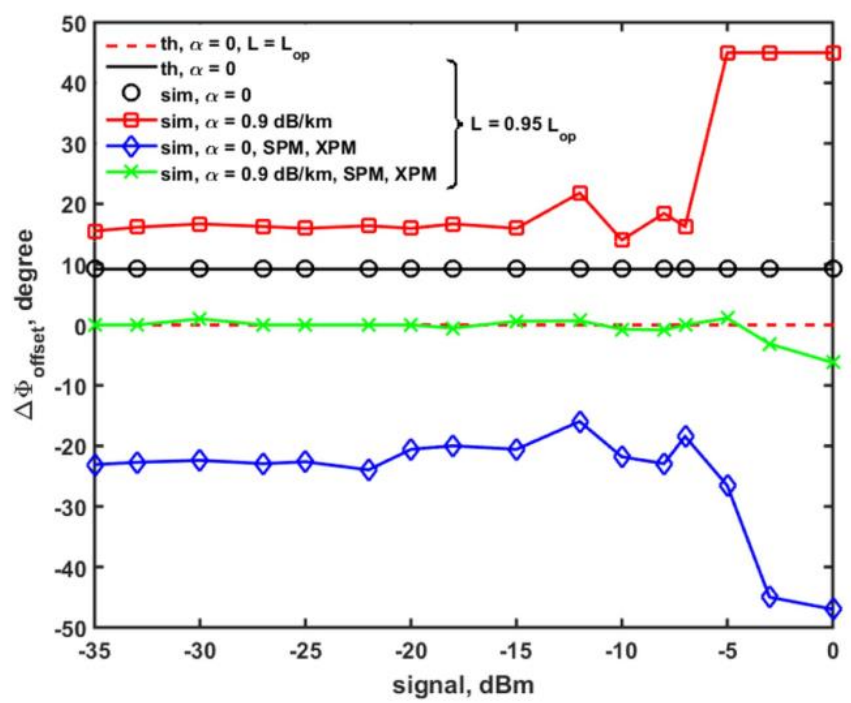

(b)

Figure 2. Effect of signal power on idler. In 2(a), efficiency and 2(b) phase shift of idler signal as a function of signal power. 


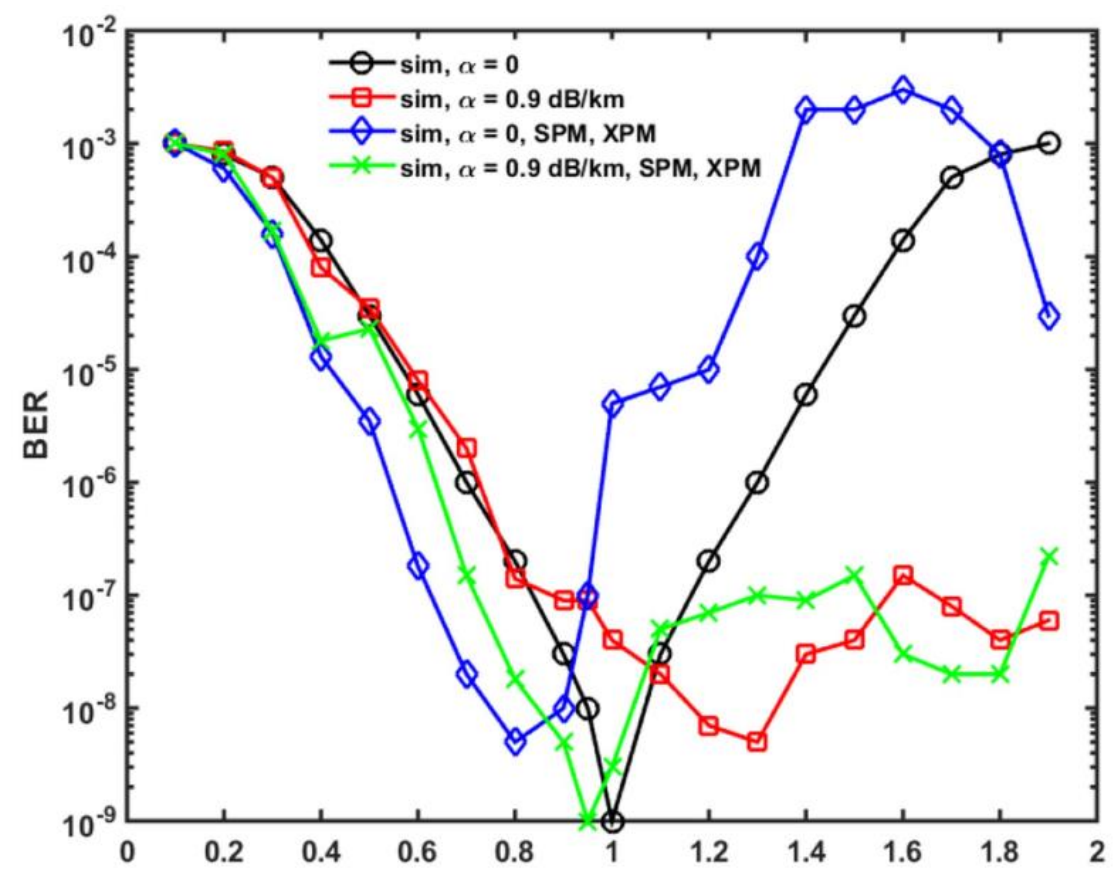

Figure 3. BER as a function of HNLF length.

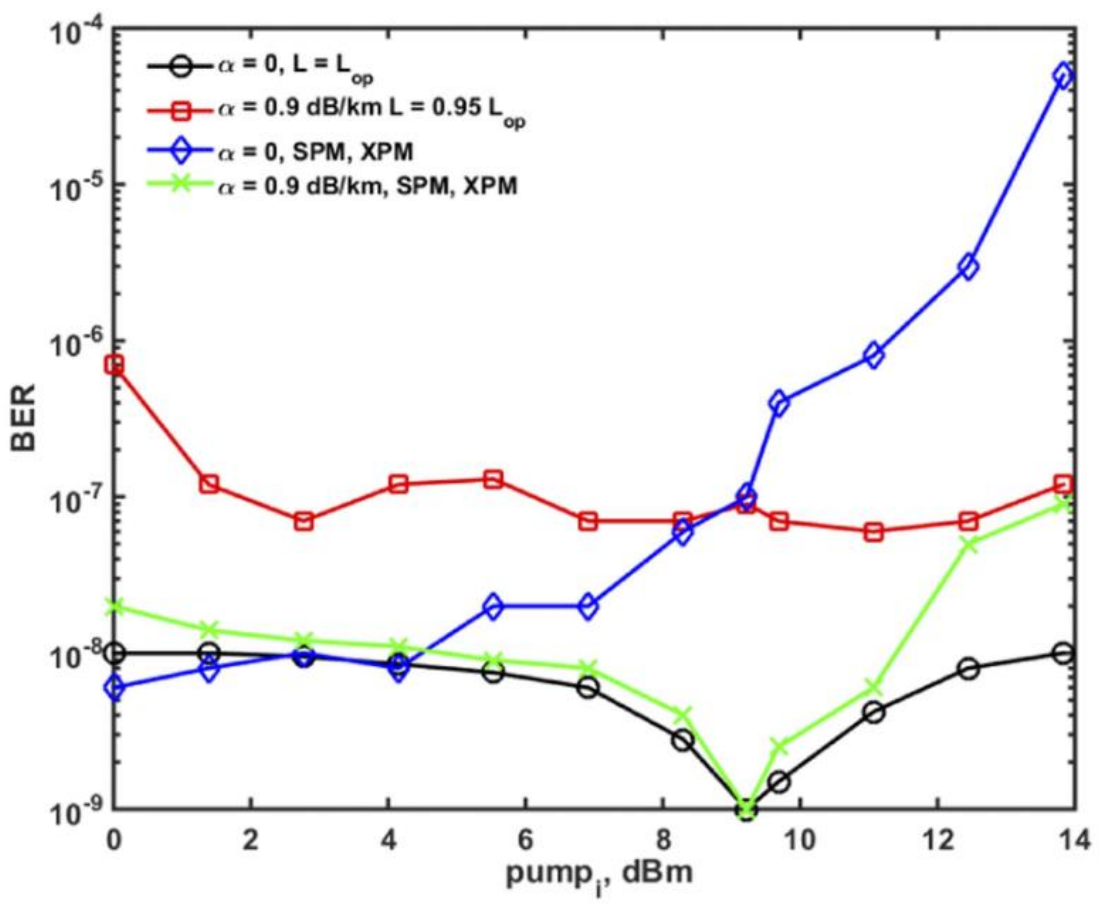

Figure 4. BER as a function of $\operatorname{pump}_{i}$ power, where $i=1$ or 2 . 


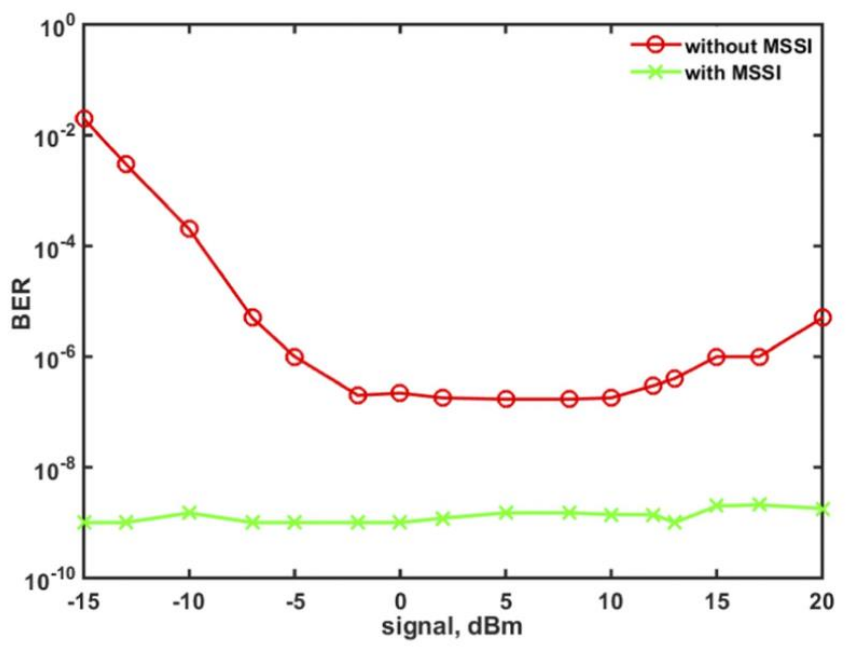

Figure 5. BER performance with signal power 


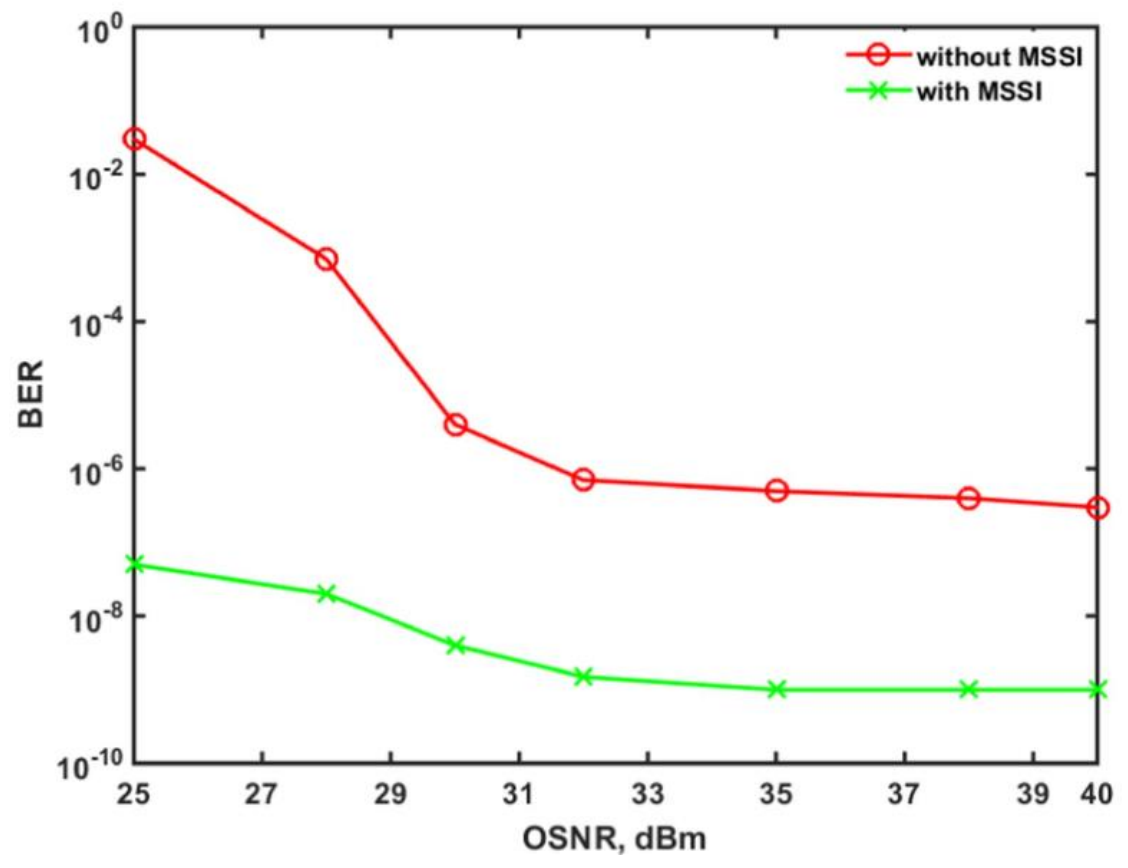

Figure 6. BER performance with transmitted OSNR. 